\title{
Effect of edaravone therapy in Korean amyotrophic lateral sclerosis (ALS) patients
}

\author{
Jin-Mo Park ${ }^{1} \cdot$ Sun-Young Kim ${ }^{2} \cdot$ Donghwi Park $^{3} \cdot$ Jin-Sung Park ${ }^{4}(D$ \\ Received: 23 April 2019 / Accepted: 24 August 2019 / Published online: 30 August 2019 \\ (C) Fondazione Società Italiana di Neurologia 2019
}

\begin{abstract}
Oxidative stress caused by free radicals has been implicated in the pathogenesis of amyotrophic lateral sclerosis (ALS). Edaravone (also known as MCI-186), a free radical scavenger, was approved as an ALS treatment in 2015 in Japan. However, the therapeutic effects of edaravone on patients with ALS outside of Japan are not yet reported. This study aims to investigate effects of edaravone on ALS patients in the Korean population. The study included 22 patients with ALS who were treated with edaravone. Of the 16 patients who finished six cycles of treatment, a mean decline of ALSFRS-R after the treatments was $5.75 \pm$ 6.07 points and the average change of FVC was $-8.7 \pm 17.0 \%$. Patients experienced only minor adverse events. This study reports on the open-label study of edaravone on patients in Korea for ALS patients, which showed a modest effect of edaravone in this population of ALS patients.
\end{abstract}

Keywords Amyotrophic lateral sclerosis (ALS) $\cdot$ Edaravone $\cdot$ Korean ALS $\cdot$ ALSFS-R

\section{Introduction}

Amyotrophic lateral sclerosis (ALS) is a lethal neurodegenerative disease characterized by gradual loss of upper and lower motor neurons, and is the most prototypic among motor neuron diseases. ALS leads to progressive muscle atrophy and weakness of the upper and lower extremities, bulbar palsy, and finally death within 3-5 years of onset due to respiratory failure. Although the exact cause of ALS remains unclear, various mechanisms including the deposition of intranuclear and cytosolic protein and RNA aggregates, disturbances of protein degradation, defective nucleocytoplasmic trafficking, mitochondrial

Jin-Sung Park

neurojspark@gmail.com; jinforeva@gmail.com

1 Department of Neurology, Dongguk University College of Medicine, Dongguk University Gyeongju Hospital, Gyeongju, South Korea

2 Department of Neurology, Ulsan University Hospital, University of Ulsan College of Medicine, Ulsan, South Korea

3 Department of Rehabilitation, Daegu Fatima hospital, Daegu, South Korea

4 Department of Neurology, School of Medicine, Kyungpook National University, Kyungpook National University Chilgok Hospital, 807 Hoguk-ro, Buk-gu, Daegu 41944, South Korea dysfunction, endoplasmic reticulum stress, altered neuronal excitability, altered axonal transport, and excessive oxidative stress have been implicated in the pathogenesis of ALS [1]. Oxidative stress might be an essential factor for the development of neurodegeneration in ALS. Several studies reported increased concentrations of the 3nitrotyrosine (3-NT) marker for oxidative stress in the spinal cord of ALS patients and increased 3-NT immunoreactivity in motor neurons of both sporadic and familial ALS patients [2]. A recent study revealed that the magnitude of oxidative stress as measured by positron emission tomography of the brain (PET scan) with $\left[{ }^{62} \mathrm{Cu}\right]$ diacetyl-bis $\left(\mathrm{N}^{4}\right.$ methylthiosemicarbazone) correlates with clinical severity in ALS [3]. A biochemical study indicated that edaravone has been shown to eliminate peroxynitrite as well as lipid peroxyl radicals [4]. A second randomized, double-blind, placebo-controlled phase 3 trial in Japan demonstrated a significantly smaller decline in ALSFRS-R score compared with placebo [5]. Based on this clinical trial, edaravone was approved as a therapeutic drug for ALS in June 2015 in Japan and by the Korean Ministry of Food and Drug Safety in December 2015. However, therapeutic effects and tolerance of edaravone on patients with ALS outside Japan are not yet reported. The aim of this study was to retrospectively investigate the effects of edaravone on ALS patients in the Korean population. 


\section{Materials and methods}

\section{Participants}

We identified twenty-two patients with ALS who were treated with edaravone between 2016 and 2018. All patients were diagnosed with ALS according to the revised El Escorial criteria $[6,7]$. Medical records were obtained after informed consent from all the patients who participated in the study. The medical records of patients who received edaravone therapy were reviewed and it was approved by the Institutional Review Board of Kyungpook National University Chilgok Hospital and Ulsan University Hospital.

\section{Methods}

Clinical parameters including age, sex, age of onset, age of diagnosis, disease duration, body weight, height, and body mass index (BMI) were retrieved from the medical records. ALSFRS-R was evaluated from the initial treatment throughout six cycles of treatment. Pulmonary function was checked with forced vital capacity (FVC) and forced expiratory volume in $1 \mathrm{~s}$ (FEV1). Patients were categorized with limb-onset type or bulbar-onset type based on the clinical features at the onset. Adverse events during six cycles of treatment were verified to determine the safety of edaravone. Edaravone was administered once per day via 60-min intravenous infusion. Edaravone was initially administered for 14 consecutive days, followed by a 2 -week drug-free period, and subsequently administered for 10 days within a 2-week period according to the standard protocol [5]. The ALSFRS-R score consisted of 12 features which we divided into four domains as follows: bulbar (items 1-3), fine motor (items 4-6), gross motor (items 7-9), and respiratory (items 10-12) [8]. We estimated the changes of ALSFRS-R score and its individual domains to ascertain therapeutic effects.

\section{Statistical analysis}

Differences in clinical characteristics between groups were analyzed using the chi-square test and/or Fisher's exact test. Comparison of differences in ALSFRS-R and its domains was analyzed by two-way ANOVA.

\section{Results}

\section{Baseline characteristics}

Initially, 22 patients (13 men, 9 women; mean age, $56.1 \pm$ 12.4 years) were treated with edaravone (Table 1 ). The average age of onset was $54.2 \pm 12.4$ years. The average disease duration was $1.9 \pm 1.2$ years. Among the patients recruited, 18 patients had a limb-onset type and four had a bulbar-onset type. Nineteen patients met definitive ALS diagnostic criteria and three patients were probable ALS [6, 7]. Fifteen patients who had been treated with riluzole continued that treatment. Only two of the 22 patients met the inclusion criteria for the second phase 3 trial: scores of $\geq 2$ points on all 12 items of the ALSFRS-R, forced vital capacity $\geq 80 \%$, definite or probable ALS according to the revised El Escorial criteria, and disease duration of $\leq 2$ years [5]. Six patients dropped out during follow-up due to adverse events such as aspiration pneumonia, abrupt clinical deterioration, and refusal of the continuous treatment. Finally, 16 patients completed six cycles of the treatment (patient 1 patient 16 in Table 1). The average onset age of these 16 patients was $52.7 \pm 9.2$ years. The duration of disease was $2.1 \pm 1.0$ years. Twelve (75\%) patients had limbonset type disease and four (25\%) patients had bulbar-onset type. The initial average score of ALSFRS-R was $34.25 \pm$ 7.22 .

\section{Changes in ALSFRS-R score, its domains, and other biologic markers}

Changes in ALSFRS-R total score in all patients are shown in Fig. 1. Among 16 patients who finished six cycles of treatment, a mean decline of ALSFRS-R after six cycles of treatment was $5.75 \pm 6.07$ points ( $34.25 \pm 7.22$ to $28.50 \pm 8.21)$. Patient 8 showed the biggest change (26 points) of ALSFRS-R and he was the only person who showed a change of more than 10 points. A mean decline of ALSFRS-R excluding patient 8 was $4.40 \pm 2.87$. In contrast, the ALSFRS-R of patient 16 was 45 points throughout the treatment period consistently. Eleven patients (68.8\%) experienced a stable course of progression (less than one-point reduction of ALSFRS-R per month) [9]. In this stable group, the mean decline was $3.00 \pm 2.05$. In contrast, the mean decline was $11.60 \pm 8.14$ in the remaining (the declined group).

For ALSFRS-R subscore, the mean decline was $0.93 \pm$ 1.14 in the bulbar domain, $1.21 \pm 2.01$ in fine motor domain, $1.57 \pm 1.50$ in gross motor domain, and $1.57 \pm 3.18$ in respiratory domain. Changes of fewer than five points were observed in 14 patients in bulbar domain, 13 in fine motor domain, 12 in gross motor domain, and 12 in respiratory domain.

A change of FVC was confirmed in six patients. Their initial mean FVC was $75.0 \pm 8.2 \%$ and declined to $66.3 \pm$ $21.6 \%$. The average change of FVC was $-8.7 \pm 17.0 \%$. The mean changes in body weight and BMI in 10 patients were $2.3 \pm 4.4$ and $-0.7 \pm 1.6$, respectively.

Patients were divided into the stable group (11 patients), in which the total score of ALSFRS-R decreased to below five points, and the declined group (5 patients). There was no statistically significant difference in onset type $(p=0.35)$, age at onset $(p=0.49)$, disease duration $(p=0.72)$, change of FVC, BMI, and score change of each domain between the 
Table 1 Baseline clinical and characteristic features of patients included in the study

$\begin{array}{llllllllllllllllllllllll}\text { Pt } 1 & \text { Pt } 2 & \text { Pt } 3 & \text { Pt } 4 & \text { Pt } 5 & \text { Pt } 6 & \text { Pt } 7 & \text { Pt } 8 & \text { Pt } & \text { Pt } & \text { Pt } & \text { Pt } & \text { Pt } & \text { Pt } & \text { Pt } & \text { Pt } & \text { Pt } & \text { Pt } & \text { Pt } & \text { Pt } & \text { Pt } & \text { Pt }\end{array}$

$\begin{array}{llllllllllllll}9 & 10 & 11 & 12 & 13 & 14 & 15 & 16 & 17 & 18 & 19 & 20 & 21 & 22\end{array}$

\begin{tabular}{|c|c|c|c|c|c|c|c|c|c|c|c|c|c|c|c|c|c|c|c|c|c|c|}
\hline Age & 47 & 44 & 55 & 65 & 53 & 47 & 51 & 45 & 30 & 52 & 63 & 60 & 63 & 64 & 51 & 53 & 35 & 76 & 67 & 69 & 80 & 64 \\
\hline Sex & $\mathrm{F}$ & F & M & M & F & $\mathrm{F}$ & M & M & M & M & M & F & M & $\mathrm{F}$ & $\mathrm{F}$ & M & $F$ & $F$ & $M$ & $M$ & $M$ & $M$ \\
\hline $\begin{array}{l}\text { Disease } \\
\text { duration }\end{array}$ & 2 & 4 & 2 & 2 & 1 & 1 & 4 & 2 & 1 & 1 & 2 & 2 & 2 & 3 & 3 & 1 & 1 & 3 & 3 & 1 & 1 & 3 \\
\hline Onset type & $\mathrm{L}$ & L & $\mathrm{L}$ & $\mathrm{L}$ & L & $\mathrm{L}$ & B & B & $\mathrm{L}$ & $\mathrm{L}$ & B & $\mathrm{L}$ & $\mathrm{L}$ & B & $\mathrm{L}$ & $\mathrm{L}$ & $L$ & $L$ & $L$ & $L$ & $L$ & $L$ \\
\hline $\begin{array}{l}\text { ALS } \\
\quad \text { diagnostic } \\
\text { criteria }\end{array}$ & $\mathrm{D}$ & $\mathrm{D}$ & $\mathrm{D}$ & $\mathrm{D}$ & $\mathrm{D}$ & $\mathrm{P}$ & $\mathrm{D}$ & $\mathrm{P}$ & $\mathrm{D}$ & $\mathrm{D}$ & $\mathrm{D}$ & $\mathrm{D}$ & $\mathrm{D}$ & $\mathrm{D}$ & $\mathrm{D}$ & $\mathrm{D}$ & $P$ & $D$ & $D$ & $D$ & $D$ & $D$ \\
\hline $\begin{array}{l}\text { Initial } \\
\quad \text { ALSFRS-R }\end{array}$ & 25 & 40 & $25^{*}$ & 38 & 31 & 27 & 42 & 42 & 40 & 30 & 29 & 28 & 27 & 44 & 35 & 45 & 44 & 27 & 32 & 41 & 30 & 38 \\
\hline $\begin{array}{l}\text { Bulbar } \\
\text { domain }\end{array}$ & 9 & 11 & - & 12 & 10 & 7 & 9 & 7 & 10 & 11 & 4 & 6 & 10 & 10 & 9 & 11 & 12 & 9 & 11 & 9 & 9 & 10 \\
\hline $\begin{array}{l}\text { Fine motor } \\
\text { domain }\end{array}$ & 2 & 8 & - & 9 & 6 & 7 & 10 & 12 & 9 & 5 & 6 & 6 & 1 & 10 & 8 & 10 & 10 & 4 & 7 & 9 & 6 & 9 \\
\hline $\begin{array}{l}\text { Gross motor } \\
\text { domain }\end{array}$ & 2 & 9 & - & 5 & 7 & 3 & 11 & 11 & 9 & 2 & 7 & 6 & 6 & 12 & 5 & 12 & 10 & 4 & 2 & 12 & 5 & 10 \\
\hline $\begin{array}{l}\text { Respiratory } \\
\text { domain }\end{array}$ & 12 & 12 & - & 12 & 10 & 10 & 12 & 12 & 12 & 12 & 12 & 10 & 10 & 12 & 9 & 12 & 12 & 10 & 11 & 11 & 10 & 9 \\
\hline FVC (\%) & - & 81 & 82 & 77 & 78 & 62 & - & - & - & 81 & 34 & 73 & 84 & 70 & 73 & 80 & 94 & 38 & & 51 & 67 & 50 \\
\hline FEV1 & - & 97 & 98 & 97 & 89 & 57 & - & - & - & - & - & - & - & 67 & 74 & 88 & & & & & & 51 \\
\hline BMI & 22.7 & 20.9 & 21.2 & 21.1 & 26.4 & 21.5 & 17.9 & 19.4 & - & 26.1 & 16.1 & 25.0 & 25.4 & 20.0 & 24.8 & 21.0 & 21.4 & 19.1 & & 17.7 & 20.3 & 24.0 \\
\hline Use of riluzole & $\mathrm{Y}$ & $\mathrm{Y}$ & $\mathrm{Y}$ & $\mathrm{Y}$ & $\mathrm{N}$ & $\mathrm{Y}$ & $\mathrm{Y}$ & $\mathrm{N}$ & $\mathrm{N}$ & $\mathrm{N}$ & $\mathrm{Y}$ & $\mathrm{Y}$ & $\mathrm{Y}$ & $\mathrm{Y}$ & $\mathrm{Y}$ & $\mathrm{Y}$ & $Y$ & $Y$ & $Y$ & $N$ & $Y$ & $Y$ \\
\hline
\end{tabular}

$L$ limb-onset type, $B$ bulbar-onset type, $D$ definite ALS, $P$ probable ALS, ALSFRS- $R$ revised ALS functional rating scale, $F V C$ forced vital capacity, FEV1 forced expiratory volume in $1 \mathrm{~s}, P A S$ Penetration Aspiration Scale, $Y$ yes, $N$ no, (-) not obtained

Patients 17 22 in italics were dropped out

*Patient 3 took the first cycle edaravone in other hospital, only the total ALSFRS-R score was gained

Fig. 1 Serial changes in ALSFRS-R total score during six cycles of treatment. These showed a variation in the response during treatments (a) and changes of mean ALSFRS-R score (b) in these 16 patients

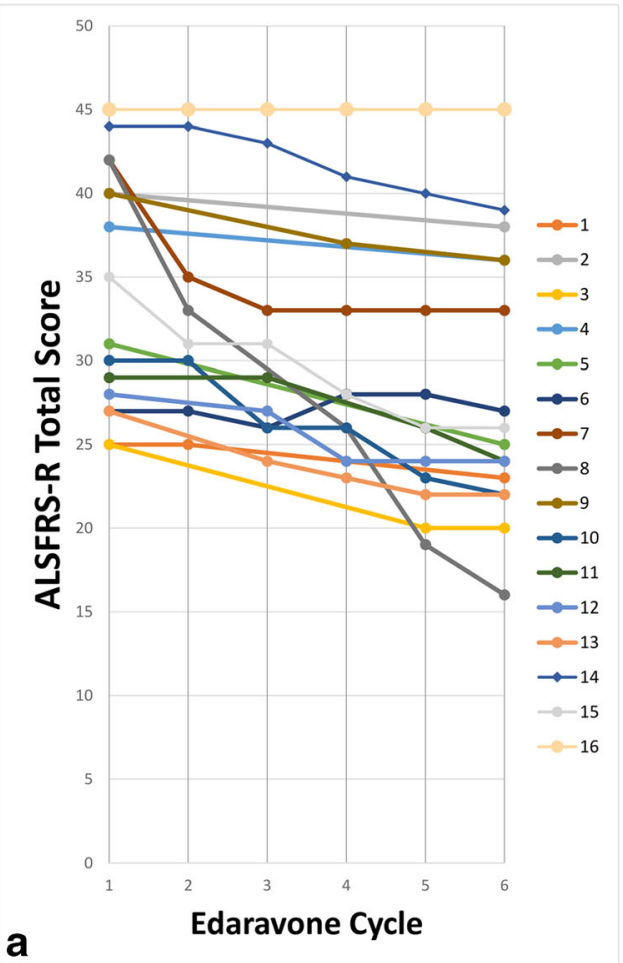


groups. There was no significant difference when patients were divided into onset types, age at onset $(p=0.57)$, disease duration ( $p=0.11$ ), changes of FVC, BMI, and score change of each domain.

\section{Adverse events}

No patients who were treated with edaravone experienced any significant adverse events that led to discontinuation of the drug. However, two patients reported skin rash during edaravone administration. Fortunately, occurrences were minor and easily tolerated with pre-treatment of chlorpheniramine and steroids. Other relatively common adverse events were constipation $(n=4)$, insomnia $(n=3)$, headache $(n=2)$, and transient leukopenia $(n=1)$. These symptoms were well tolerated with appropriate medications to treat symptoms.

Discontinuation of edaravone treatment occurred in 6 patients. In patient 20 , aspiration pneumonia occurred during edaravone treatment. He was treated in the intensive care unit and was excluded from the analysis due to the delayed treatment schedule. Pneumonia may have been a symptom of ALS rather than an adverse event of edaravone. Another patient dropped out due to accidental disconnection of a ventilator leading to an abrupt clinical deterioration. Four patients changed their minds and decided to decline edaravone treatment.

Percutaneous endoscopic gastrostomy was applied in patient 8 (cycle 4) and 11 (cycle 3), both of whom finished six cycles of treatment. Sixteen patients who finished six cycles of edaravone treatment did not use noninvasive positive pressure ventilation or receive tracheostomy. Primary spontaneous pneumothorax also did not occur in these 16 patients [10].

\section{Discussion}

In this observational study, ALS patients on edaravone treatment showed modest results in ALSFRS-R and pulmonary function tests. Several studies of edaravone have reported significant outcomes in ALS treatment. Recently, long-term (18month) effects of edaravone were favorable in ALSFRS-R score and survival rates [11]. A recent meta-analysis study of 183 patients who received edaravone showed an efficacious result without significant adverse events, consolidating the clinical evidence with the usage of edaravone [12].

ALS researchers have evaluated disease progression using ALSFRS-R. They have observed declines in ALSFRS-R score with disease progression at a rate that is quite consistent across clinical trials: -0.92 units per month with a relatively small variance (standard error of 0.08) in the Northeast ALS Consortium database [9]. A recent meta-analysis of demographic and clinical data from Japanese, US, and European studies also showed a similar decline of 1.03 to 1.21 points/ month in Japanese patients and 0.89 to 1.60 points/month in Caucasians. This suggests that the findings of these studies can be generalized in ALS patients irrespective of ethnicity [13]. The phase 3 clinical trial on edaravone showed an ALSFRS-R decline of mean 5.01 points in 6 months in the treated group, and a mean decline of 7.50 points in 6 months in the control group showing a difference of 2.49 points at the end of 6 months with statistical significance [5]. Our study showed a mean decline of 5.75 points which are in accordance with the edaravone treated group of the recent phase III clinical study. The baseline characteristics of the ALS patients enrolled in this study had a mean ALSFRS-R of 34.25 and mean FVC of $75 \%$, reflecting a more advanced stage of ALS patients in these study patients compared with the recent phase 3 clinical trial. It is noteworthy to mention that a recent study on advanced ALS patients with a FVC of less than $60 \%$ showed no benefit of edaravone [14], reflecting the importance of early intervention in treating ALS patients, and our study showed significant efficacy in ALS patients with a mean FVC of $75 \%$. More studies on different stages of ALS patients are needed to tailor down treatment criteria of edaravone.

Recent studies have shown a modest decline in all four domains of ALSFRS-R: bulbar, fine motor, gross motor, and respiratory; interestingly, these studies resulted in a similar prognosis in both bulbar and limb-onset ALS [11]. In our study, results also indicate that reduction is not limited to a specific domain but is even across various domains of ALSFRS-R. Of the 12 limb-onset patients and four bulbaronset patients in our study, patients showed a statistically insignificant mean decline of $3.92 \pm 10.01$ and $11.25 \pm 2.86$ in the ALSFRS-R, respectively. Castillo-Viguera et al. suggested that a suppression of ALSFRS-R by more than $20 \%$ is clinically significant [15]; the phase 3 clinical trial on edaravone showed a suppression of $33 \%$, similar to our study which showed suppression of $23 \%$ at the end of 6 months. When compared by gender, onset site or stable/declined groups, there were no statistical differences.

We also evaluated pulmonary function. The phase 3 clinical trial showed a $15.61 \%$ decrease in FVC after 6 months. Our study patients showed an $8.7 \%$ decrease in FVC after 6 months, consistent with previous results. However, these results were obtained in only in six patients, only three of whom with an FVC of 80 or greater. It is noteworthy that patient 8 experienced a rapid decline in ALSFRS-R despite edaravone treatment. The rapid deterioration may have resulted from a variety of ALS phenotypes, but further studies are needed to better elucidate appropriate criteria for edaravone treatment [16].

Edaravone is known to result in frequent adverse events, ranging to $84 \%$. The most common adverse events are contusion, constipation, contact dermatitis, dysphagia, eczema, and upper respiratory tract inflammation (in order of descending frequency); $16 \%$ of the patients experienced serious adverse 
events. In our study, two patients experienced eczema and pruritis, which were well tolerated with anti-histamine and oral steroid treatment. It is also noteworthy to state transient leukopenia in one patient who recovered after a few days of initial treatment. No death was encountered during the followup period.

Limitations of our study are as follows. The study was observational, with no control group for comparison. The modest number of recruited patient needs to be considered in evaluating the results. Further studies with more patients are needed to fully understand the effect of edaravone in different stages of ALS.

\section{Conclusions}

This is a study in Korean patients to report on the open-label study of edaravone on ALS patients. The treatment was well tolerated without significant adverse events. In accordance with previous studies conducted in Japan, the US, and Europe, our study showed no difference across ethnicities in disease progression in relation to uniform oxidative stress. Our study is of clinical significance as the treatment was well tolerated and showed modest improvement in a more advanced stage of ALS. More studies from various countries are warranted to confirm the effect of edaravone in patients with ALS in various stages.

Funding information This work was supported by the National Research Foundation of the Republic of Korea, Ministry of Science and ICT (No. 2017R1C1B5076264 and NRF-2018R1C1B5045675).

Compliance with ethical standards This study was approved by the Institutional Review Board of Kyungpook National University Chilgok Hospital and Ulsan University Hospital.

Competing interests The authors declare that they have no competing interests.

\section{References}

1. Brown RH, Al-Chalabi A (2017) Amyotrophic lateral sclerosis. N Engl J Med 377:162-172

2. Beal MF, Ferrante RJ, Browne SE, Matthews RT, Kowall NW, Brown RH (1997) Increased 3-nitrotyrosine in both sporadic and familial amyotrophic lateral sclerosis. Ann Neurol 42:644-654

3. Ikawa M, Okazawa H, Tsujikawa T et al (2015) Increased oxidative stress is related to disease severity in the ALS motor cortex: a PET study. Neurology 84:2033-2039
4. Fujisawa A, Yamamoto Y (2016) Edaravone a potent free radical scavenger, reacts with peroxynitrite to produce predominantly 4NO-Edaravone. Redox Rep 21:98-103

5. Writing Group; Edaravone (MCI-186) ALS 19 Study Group (2017) Safety and efficacy of edaravone in well defined patients with amyotrophic lateral sclerosis: a randomised, double-blind, placebo-controlled trial. Lancet Neurol 16:505-512

6. Brooks BR, Miller RG, Swash M, Munsat TL (2000) World Federation of Neurology Research Group on motor neuron D. El Escorial revisited: revised criteria for the diagnosis of amyotrophic lateral sclerosis. Amyotroph Lateral Scler Other Motor Neuron Disord 1:293-299

7. Amin Lari A, Ghavanini AA, Bokaee HR (2019) A review of electrophysiological studies of lower motor neuron involvement in amyotrophic lateral sclerosis. Neurol Sci 40:1125-1136

8. Bacci ED, Staniewska D, Coyne KS, Boyer S, White LA, Zach N, Pooled Resource Open-Access ALS Clinical Trials Consortium et al (2016) Item response theory analysis of the amyotrophic lateral sclerosis functional rating scale-revised in the pooled resource open-access ALS clinical trials database. Amyotroph Lateral Scler Frontotemporal Degener 17:157-167

9. Castrillo-Viguera C, Grasso DL, Simpson E, Shefner J, Cudkowwicz ME (2010) Clinical significance in the change of decline in ALSFRS-R. Amyotroph Lateral Scler 11:178-180

10. Park JS, Do YW, Park JM, Seok HY, Park D (2019) Underrecognized primary spontaneous pneumothorax in ALS: a multicenter retrospective study. Neurol Sci. https://doi.org/10.1007/ s10072-019-03989-y

11. Okada M, Yamashita S, Ueyama H, Ishizaki M, Maeda Y, Ando Y (2018) Long-term effects of Edaravone on survival of patients with amyotrophic lateral sclerosis. eNeurologicalSci 11:11-14

12. Luo L, Song Z, Li X et al (2018) Efficacy and safety of edaravone in treatment of amyotrophic lateral sclerosis - a systemic review and meta-analysis. Neurol Sci 40:235-241

13. Takei K, Takahashi F, Liu S, Tsuda K, Palumbo J (2017) Post-hoc analysis of randomised, placebo-controlled, double-blind study (MCI186-19) of Edaravone (MCI-186) in amyotrophic lateral sclerosis. Amyotroph Lateral Scler Frontotemporal Degener 18:49-54

14. Writing group on behalf of the edaravone (MCI-186) ALS 18 study group (2017) Exploratory double-blind, parallel-group, placebocontrolled study of edaravone (MCI-186) in amyotrophic lateral sclerosis (Japan ALS severity classification: grade 3, requiring assistance for eating, excretion or ambulation). Amyotroph Lateral Scler Frontotemporal Degener 18:40-48

15. Takei K, Tsuda K, Takahashi F, Hirai M, Palumbo J (2017) An assessment of treatment guidelines, clinical practices, demographics, and progression of disease among patients with amyotrophic lateral sclerosis in Japan, the United States, and Europe. Amyotroph Lateral Scler Frontotemporal Degener 18:88-97

16. Watanabe H, Atsuta N, Hirakawa A, Nakamura R, Nakatochi M, Ishigaki S, Iida A, Ikegawa S, Kubo M, Yokoi D, Watanabe H, Ito M, Katsuno M, Izumi Y, Morita M, Kanai K, Taniguchi A, Aiba I, Abe K, Mizoguchi K, Oda M, Kano O, Okamoto K, Kuwabara S, Hasegawa K, Imai T, Kawata A, Aoki M, Tsuji S, Nakashima K, Kaji R, Sobue G (2016) A rapid functional decline type of amyotrophic lateral sclerosis is linked to low expression ofTTN. J Neurol Neurosurg Psychiatry 87:851-858

Publisher's note Springer Nature remains neutral with regard to jurisdictional claims in published maps and institutional affiliations. 